\title{
Interstitial pressure and lung oedema in chronic hypoxia
}

\author{
I. Rivolta*, V. Lucchini" ${ }^{\#}$ M. Rocchetti ${ }^{\uparrow}$, F. Kolar ${ }^{+}$, F. Palazzo \\ A. Zaza" and G. Miserocchi*
}

ABSTRACT: We evaluated how the increase in lung interstitial pressure correlates with the pulmonary vascular response to chronic hypoxia.

In control and hypoxic (30 days; $10 \% \mathrm{O}_{2}$ ) Wistar male rats, we measured: pulmonary interstitial pressure $\left(P_{i p}\right)$, cardiac and haemodynamic parameters by echocardiography, and performed lung morphometry on tissue specimens fixed in situ.

In control animals, mean \pm SD $P$ ip, air/tissue volume ratio and capillary vascularity index in the air-blood barrier were $-12 \pm 2.03 \mathrm{cmH}_{2} \mathrm{O}, 3.9$ and 0.43 , respectively. After hypoxia exposure, the corresponding values of these indices in apparently normal lung regions were $2.6 \pm 1.7 \mathrm{cmH}_{2} \mathrm{O}$, 3.6, and 0.5, respectively. In oedematous regions, the corresponding values were $12 \pm 4 \mathrm{cmH}_{2} \mathrm{O}$, 0.4 and 0.3 , respectively. Furthermore, in normal regions, the density of pre-capillary vessels (diameter $\sim$ 50-200 $\mu \mathrm{m}$ ) increased and their thickness/internal diameter ratio decreased, while opposite results were found in oedematous regions. Pulmonary artery pressure increased in chronic hypoxia relative to the control (39.8 \pm 5.9 versus $26.2 \pm 2.2 \mathrm{mmHg})$.

Heterogeneity in local lung vascular response contributes to developing pulmonary hypertension in chronic hypoxia. In oedematous regions, the decrease in capillary vascularity correlated with the remarkable increase in interstitial pressure and morphometry of the pre-capillary vessels suggested an increase in vascular resistance; the opposite was true in apparently normal regions.

KEYWORDS: Capillary patency, lung morphometry, microvascular permeability, pulmonary hypertension, pulmonary interstitial pressure

$\mathrm{T}$ he present study reports the first in situ measurements of peri-microvascular pulmonary interstitial pressure $(P$ ip $)$ in lungs exposed to chronic hypoxia, a condition that characteristically entrains a marked perturbation in interstitial fluid dynamics due to increased microvascular permeability [1] and vascular resistances, leading to pulmonary hypertension. These measurements allow us to discuss the impact of the tissue-capillary mechanical interaction and interstitial fluid dynamics on the pulmonary vascular response.

\section{METHODS}

Wistar male rats, age 5 weeks, were exposed for 1 month to normobaric hypoxia $\left(10 \% \mathrm{O}_{2}\right)$. Gas mixture inflow allowed keeping inspired oxygen tension at $10 \%$ (feedback control) and $\mathrm{CO}_{2}$ concentration within 1,000-1,400 ppm (0.1-0.14\%). At the end of the exposure period, several protocols were applied both to hypoxic and normoxic control rats matched for age.

Animal experimentation was performed according to the Helsinki convention for the use and care of animals.

\section{Anaesthesia}

Animals were anaesthetised (intraperitoneal 50\% diluted urethane; $1,200 \mathrm{mg} \cdot \mathrm{kg}^{-1}$ ) and placed in a supine position.

\section{Measurement of Pip}

We opened two to three "pleural windows" either on the right or the left side of the chest, allowing viewing portions of the upper or of the lower lobe, following a technique described previously and routinely adopted in our laboratory [2]. Measurements were performed in the subpleural layer of the lung, up to a depth of $\sim 50 \mu \mathrm{m}$.

\section{Measurement of peak right ventricular pressure}

A cannula was advanced through the jugular vein into the right ventricle to measure peak ventricular pressure, which was assumed equal to systolic pulmonary artery pressure $(P$ pa). A blood sample was taken to determine haematocrit $(\mathrm{Ht})$.

\section{Echocardiography}

Echocardiography was performed in seven control and seven hypoxic rats under ketamine/

\section{AFFILIATIONS}

*Dept of Experimental Medicine,

University of Milano Bicocca,

\#Anatomic Pathology, San Gerardo

Hospital, Monza

"Dept of Biotechnology and

Bioscience, University of Milano

Bicocca,

${ }^{\text {s}}$ Prassis Istituto di Ricerche Sigma-

tau, Settimo Milanese, Milan, Italy.

${ }^{+}$Institute of Physiology, Academy of

Sciences of the Czech Republic,

Prague, Czech Republic.

\section{CORRESPONDENCE}

I. Rivolta

Dept of Experimental Medicine

University of Milano Bicocca

Via Cadore, 48

20052 Monza

Italy

E-mail: ilaria.rivolta@unimib.it

Received:

April 282010

Accepted after revision:

July 112010

First published online:

Aug 062010 
xylazine $\left(130 / 5 \mathrm{mg} \cdot \mathrm{kg}^{-1}\right)$ anaesthesia after $1 \mathrm{month}$ of exposure to hypoxia. M-mode measures of right ventricle free wall thickness, and Doppler signals of the pulmonary artery were obtained using a Sequoia 512 echographer equipped with a 13-MHz probe (Acuson, Mountain View, CA, USA) in a parasternal short axis.

\section{Tissue sampling}

The trachea was tied in the neck to preserve the physiological lung expansion after chest opening. We next opened the chest, and removed the heart and lungs together.

Lungs were tied at the hylum (leaving a thread end $\sim 2 \mathrm{~cm}$ long) and put in a tube containing $4 \%$ paraformaldehyde. In order to avoid the problem of lung floating, we screwed the cap on the tube together with the thread end and then turned the tube upside down, so that lungs were floating up and pulling on the thread. The length of the thread allowed the lung to be kept fully immersed in the fixative. This fixation procedure was intended to preserve the in vivo tissuemicrovessel interaction. We excluded using a perfusion fixation procedure, as it would have considerably altered the vascular transmural pressure, which has a profound influence on vessels with highly deformable walls, such as the capillaries. Furthermore, perfusion fixation is problematic when pulmonary hypertension is present with marked regional differences in vasomotion. Histological sections were subsequently obtained, processed according to standard methods and stained with haematoxylin-eosin.

\section{Technique for morphometric evaluation}

Lung morphometry was assessed using histological sections obtained from control and hypoxic lungs (as well as on lung portions where micropuncture was performed) according to standardised techniques [3] using an Olympus BX51 microscope (Olympus, Milan, Italy). With a magnification of $400 \times$, we estimated the volume fraction of air, tissue (septa plus corners) and red blood cells using a transparent grid with $12 \times 18$ points (total points 216 ) regularly spaced at $0.8-\mathrm{cm}$ intervals fixed on the screen connected to the microscope. We analysed five images from the same specimen by counting the number of grid points falling on the air spaces, on the tissue and on the red blood cells. The number of points falling in each compartment, divided by 216 , yielded the relative volume of the given compartment.

We also determined the thickness of the alveolar septa using CellR (Olympus) software for morphometric analysis.

Finally, we estimated the capillary vascularity index (CVI) in the air-blood barrier (at a magnification of $600 \times$ ) by counting the number of red blood cells in the alveolar septa and corners divided by tissue volume. We assumed CVI to be proportional to the density of red blood cells; in order to correct for the increase in blood Ht in hypoxia, we multiplied CVI by the ratio of Htcontrol/Hthypoxia.

We also estimated the density of the pre-capillary vessels grouped into four ranges of internal diameter: <50, 51-100, 101-200, $>200 \mu \mathrm{m}$. We used a magnification of $10 \times$ for diameters $<100 \mu \mathrm{m}$, while for larger vessels we used a magnification of $4 \times$. We also expressed the density of the vessels relative to the volume of lung tissue. To do so, for each diameter group, we divided the total number of vessels counted over the images analysed by the overall surface area of the same images and further divided this ratio by the relative tissue volume.

The thickness of the pre-capillary vessel walls was estimated for the internal diameter groups $<30,31-50,51-100,101-200$, $>200 \mu \mathrm{m}$, using the same magnification specified above. We acknowledge that morphometry of pulmonary vessels provides indications concerning the geometry and extension of the vascular bed and, thus, an indirect estimate of vascular resistances. It was assumed that the latter should affect, in turn, regional blood flow.

\section{Statistical analysis}

Statistical analysis were carried out by nonparametric Pearson test and the significance level was set at $\mathrm{p}<0.01$. Data are presented as mean $\pm \mathrm{SD}$.

\section{RESULTS}

\section{Pip}

The pleural window allows a clear view the morphology of the underlying lung through a transparent visceral pleura. In hypoxic rats, we could clearly identify lung regions that retained a fairly normal morphology, thus appearing nice and pink; we designated them "well-adapted" (WA). Conversely, other regions appearing poorly aerated, congested and reddish were defined as "maladapted" (MA). Micropuncture was also performed in areas displaying transitional features between WA and MA.

Table 1 reports $P$ ip data measured in control and chronic hypoxic animals; in the latter, data were grouped for WA and MA regions, while data from transitional zones were considered as MA. As can be seen, Pip was remarkably subatmospheric in control animals and increased considerably in hypoxic rats: slightly above $0 \mathrm{cmH}_{2} \mathrm{O}$ in WA and remarkably more in MA regions. Note the increase in coefficient of variation in MA, accounting for the existence of transition states from WA to MA conditions.

\section{Systolic Ppa}

Upon exposure to chronic hypoxia, mean \pm SD systolic $P$ pa increased significantly from $26.2 \pm 2.2$ to $39.8 \pm 5.9 \mathrm{mmHg}$ $(\mathrm{n}=26$ in both groups; $\mathrm{p}<0.01)$ and $\mathrm{Ht}$ increased from $45.6 \pm 3.2$ to $52.2 \pm 3.2$.

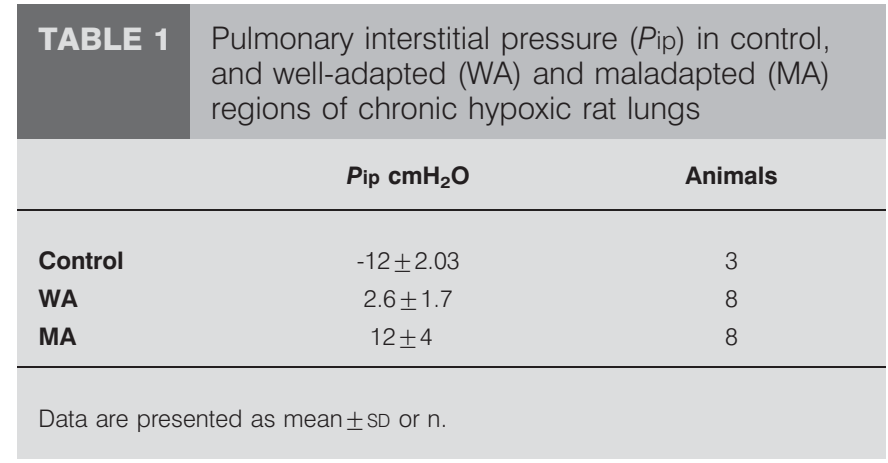




\section{Echocardiography}

After hypoxia exposure, peak blood flow velocity occurred earlier in systole and late systolic notching was often present, both representing an index of increase in $P$ pa. In hypoxic animals, mean $\pm S D$ pulmonary artery acceleration time decreased significantly from $34 \pm 7.6$ to $21 \pm 2.6 \mathrm{~ms}(\mathrm{p}<0.01)$ and the right ventricular wall thickness increased significantly from $0.44 \pm 0.10$ to $0.85 \pm 0.15 \mathrm{~mm}(\mathrm{p}<0.01)$. Cardiac output did not change significantly $\left(88 \pm 23\right.$ versus $\left.94 \pm 28 \mathrm{~mL} \cdot \mathrm{min}^{-1}\right)$.

\section{Lung morphometry}

Figure 1 shows a comparison at low magnification the homogeneity of lung morphology in a control rat (fig. 1a) as opposed to heterogeneity of lungs from a rat exposed to chronic hypoxia for 1 month (fig. 1b). In the latter case, apparently normal regions (WA) coexist with regions showing tissue congestion (MA), mostly localised in the upper lobe. The image also shows transition states between WA and MA regions. The location of MA regions was variable, including peripheral lung portions, as well as the subpleural regions. All hypoxic rats showed this bimodal pattern, although the volume fraction of MA to WA regions within the same animal was highly variable and could only be estimated roughly, based on a number of serial sections.

Relative to control (fig. 1c), lung morphology at a higher magnification in the WA regions (fig. 1d) displayed two specific features: alveolar septa appeared thinner, while alveolar corners were enlarged, vascularised and oedematous. In MA regions (fig. 1e), the features of the normal lung were completely lost; in particular, the septa could no longer be distinguished, and a marked hyperplasia of epithelial cells and accumulation of extravascular fluid was observed. Rare macrophages or inflammatory cells were found.

The relative volume of air $(A)$ (fig. 2a) in WA regions was essentially equal to control (mean \pm SD $0.65 \pm 0.04$ and $0.61 \pm 0.04$, respectively), while in MA regions it decreased significantly $(0.24 \pm 0.06 ; \mathrm{p}<0.01$ for control or WA versus MA). The relative lung tissue volume $(T)$ in WA regions was also similar to control $(0.16 \pm 0.04$ in control and $0.18 \pm 0.04$ in WA); however, this occurred due to a significant increase in relative volume of corners at the expense of a corresponding decrease in the volume of septa. In fact, the mean \pm SD thickness of the septa decreased from $4.37 \pm 0.42 \mu \mathrm{m}(\mathrm{n}=219)$ in control to $1.76 \pm 0.17 \mu \mathrm{m}(\mathrm{n}=203)(\mathrm{p}<0.01)$ in WA regions. In regions transitional between WA and MA, we were still able to identify septa and their thickness was $7.78 \pm 2.7 \mu \mathrm{m}(\mathrm{n}=179, \mathrm{p}<0.01)$, which was significantly higher than in WA regions. In MA regions, the relative tissue volume increased to 0.58 . The $A / T$ ratio decreased slightly in WA relative to control (3.6 versus 3.8 , respectively), while the septa/corners volume ratio was 4.7fold less (0.65 in WA and 3.09 in control). In MA regions, the $A / T$ ratio was dramatically reduced to 0.42 (fig. $2 b$ ). Figure $2 a$ also shows the volume component of blood, as estimated from the density of red blood cells. In controls, the relative volume of red blood cells amounts to $8 \%$ of the total lung volume, corresponding to $44 \%$ of the tissue. In the WA regions, the relative volume of blood increases up to $14 \%(p<0.01$ versus controls), corresponding to $73 \%$ of the tissue. Finally, in MA regions, the relative volume of blood was $17 \%(\mathrm{p}<0.01$ versus controls, not significantly different from WA), corresponding to $29 \%$ of tissue.

CVI was augmented in WA relative to control (fig. 3) and this occurred through a significantly greater CVI value in the corner vessels $(0.105 \pm 0.04$ versus $0.033 \pm 0.04$ in WA and control, respectively; $\mathrm{p}<0.01$ ), while the CVI of the septal vessels remained essentially the same $(0.395 \pm 0.01$ versus $0.386 \pm 0.03$ in WA versus control). In MA regions, CVI value was decreased by $31 \%$ compared with control $(0.21 \pm 0.01$ versus $0.42 \pm 0.05 ; \mathrm{p}<0.01)$.

Comparing the density of the pre-capillary vessels belonging to the four classes, as indicated on the $x$-axis of figure $4 \mathrm{a}$, as expected, it increases remarkably with decreasing internal calibre. When compared with control, WA regions showed a remarkable decrease in density of vessels in $51-100 \mu \mathrm{m}$ in diameter, but a significant increase in density of vessels with internal calibre $>100 \mu \mathrm{m}$. In MA regions, a considerable decrease in density for all vessel groups was observed.

The correlation between the thickness of the vessel wall and the internal diameter is shown in figure $4 \mathrm{~b}$, showing that the relationship is displaced downward in WA and upward in $\mathrm{MA}$, relative to control.

\section{DISCUSSION}

The present study shows that exposure of rats to chronic hypoxia causes considerable regional modifications in microvascular-interstitial fluid dynamics, capillary-tissue mechanical interaction, lung morphology and geometry of the vascular tree. In fact, a remarkable heterogeneity in the lung response was observed and, furthermore, the extremes of lung adaptation, namely WA and MA regions, coexisted within the same animal.

\section{Interstitial pressure, fluid dynamics and lung morphology}

Under physiological conditions, the volume of the extravascular water is minimal [4], so as to assure a low thickness of the air-blood barrier $(\sim 0.3 \mu \mathrm{m}$ for the thin portion). Perfect adherence of the capillary wall to the alveolar barrier is guaranteed by the rather subatmospheric pulmonary interstitial pressure [4], $\sim-12 \mathrm{cmH}_{2} \mathrm{O}$ in control rats, which reflects the balance between the powerful action of the draining lymphatic pump in face of the low permeability of the endothelial barrier [2]. Hypoxia is a known cause of perturbation of the interstitial fluid dynamic steady state condition: indeed, it increases water and solute microvascular permeability by inducing fragmentation of the extracellular matrix and, furthermore, promotes capillary recruitment, thus increasing the overall microvascular filtration surface $[1,5-8]$.

In WA regions, interstitial pressure increased up to $\sim 3 \mathrm{cmH}_{2} \mathrm{O}$, a condition similar to that previously found on acute hypoxia exposure and defined as interstitial lung oedema [1]. The remarkable increase in interstitial pressure, from -12 to $3 \mathrm{cmH}_{2} \mathrm{O}$, reflects the high elasticity of the interstitial matrix provided by the mechanical resistance of proteoglycans to the increase in tissue parenchymal stresses $[4,5]$. In terms of Starling balance of pressures across the endothelial wall, the increase in interstitial pressure represents an efficient "tissue safety factor" that counteracts the increase in filtration rate [1]. Furthermore, WA regions retain another interesting feature already described 

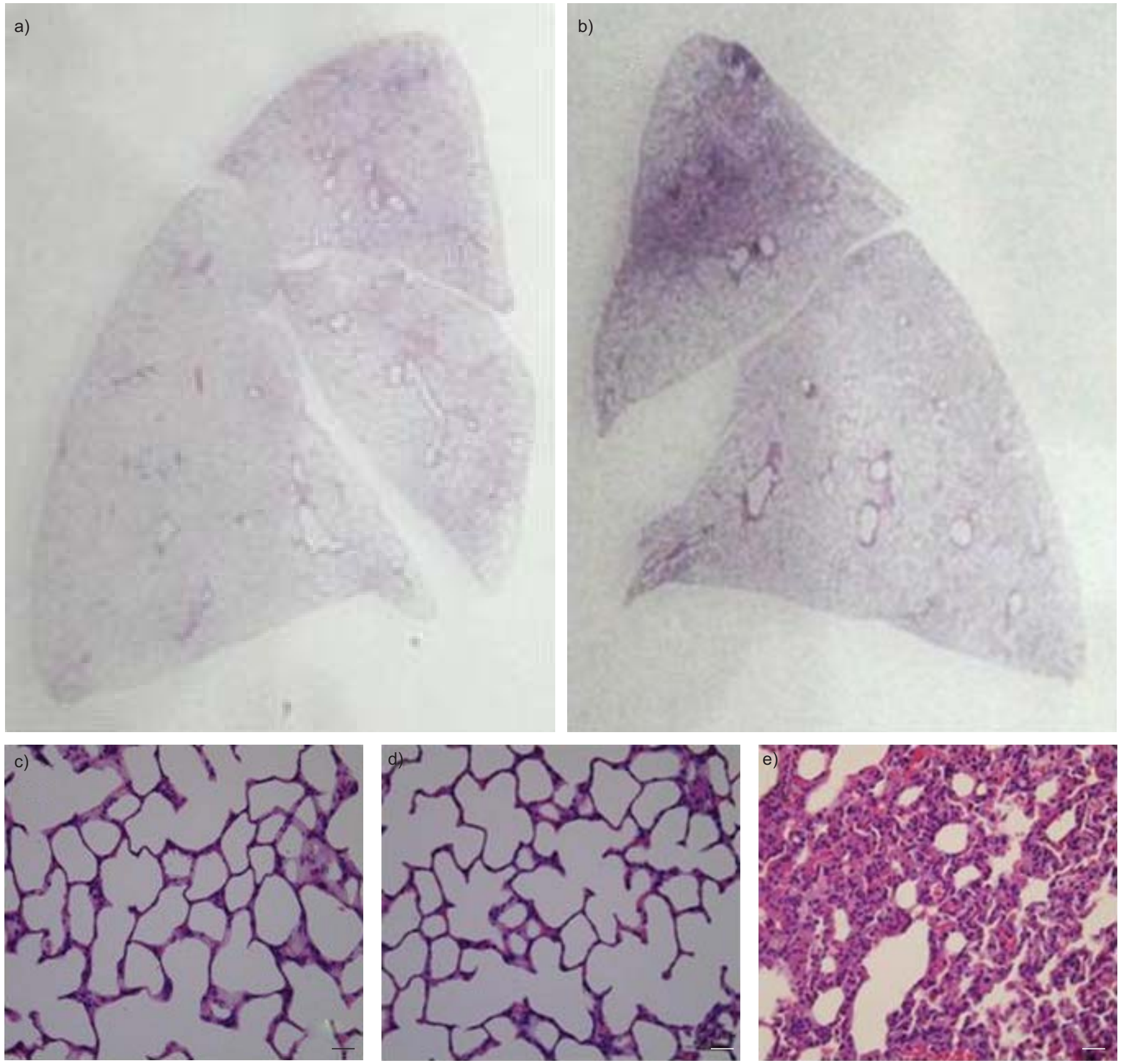

FIGURE 1. a) Low-magnification image of histological sections of a control lung to show a quite homogeneous morphology. b) Lung from a chronic hypoxic rat showing regions that appear normal (well-adapted; WA) as well as a dark region in the upper lobe that appears infiltrated (maladapted; MA). c) Histological section at $400 \times$ of a control animal. d) Histological section at $400 \times$ of a WA region: morphometric evaluation showed thinning of alveolar septa and enlarged, highly vascularised and oedematous alveolar corners in hypoxic rats. e) Histological section of MA region showing complete loss of normal lung morphology due to thickening of the septa, deposition of interstitial matrix, accumulation of extravascular fluid and marked hyperplasia of epithelial cells in a hypoxic rat. Scale bars $=30 \mu \mathrm{m}$.

for exposure to acute hypoxia [9], namely the decrease in thickness of the air-blood barrier in the septa due to the reduction of the cytoplasm volume of alveolar epithelial and endothelial cells, as determined on transmission electronic microscopy [9-11].

Interstitial oedema ought to be considered an early stage of severe oedema that was shown to develop when the extracellular matrix looses its integrity due to fragmentation of the proteoglycan components beyond a critical threshold [4-6]. Hypoxia may indeed induce matrix fragmentation by activation of tissue metalloproteases [1]. Therefore, initial interstitial oedema may have progressed to frank oedema in MA zones, occurring together with a marked alteration in tissue morphology, hyperplasia of epithelial cells and an increase in interstitial pressure similar to that reported for other oedematous tissues, such as solid tumours and brain oedema [12, 13]. One cannot exclude that in the lung the increase in tissue pressure might 

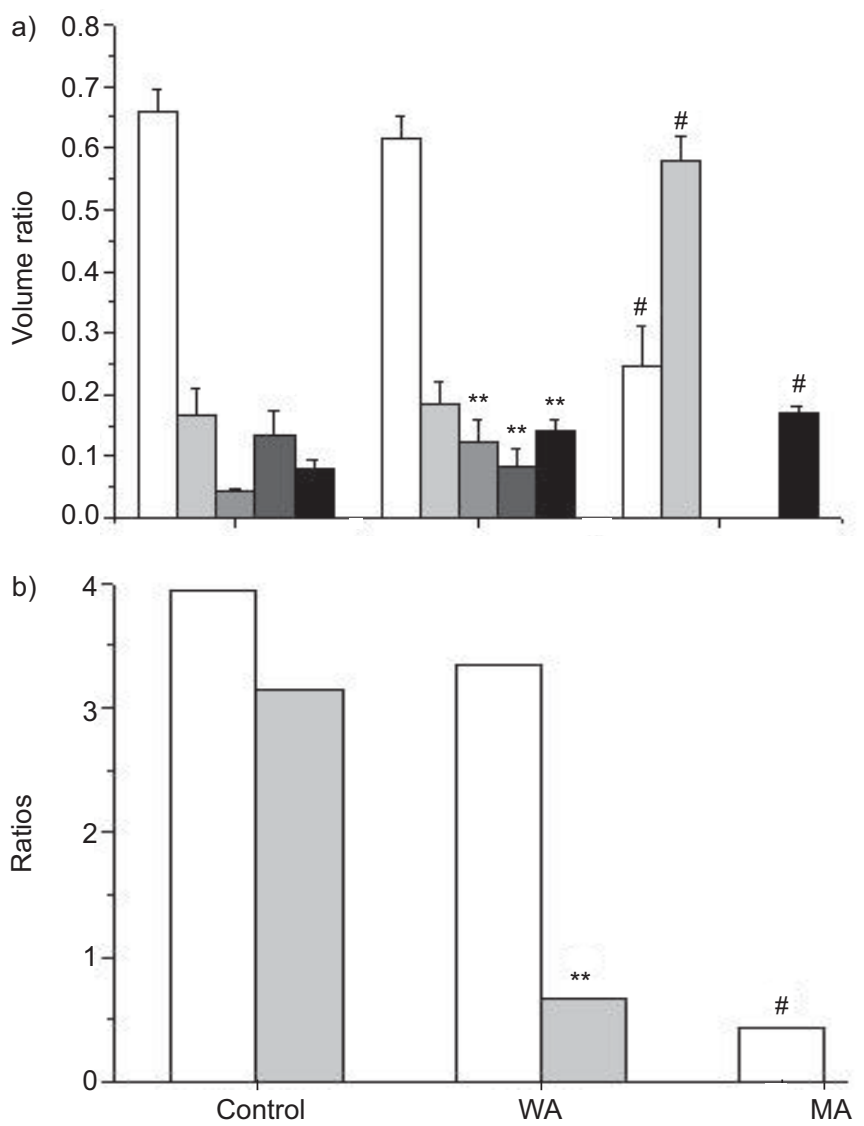

FIGURE 2. a) Relative volume of air $(\square)$, whole lung tissue $(\square)$, alveolar corners $(\square)$, alveolar septa ( $\square$ ) and red blood cells ( $\mathbf{\square})$ in control, well-adapted (WA) and maladapted (MA) regions. b) Changes in air/tissue $(\square)$ and septa/corners ( $\square$ ) volume ratios. ${ }^{* *}: p<0.01$ relative to control; ${ }^{*}: p<0.01$ relative to control and WA.

also reflect an increase in alveolar pressure (auto-PEEP) that should not exceed 1-2 $\mathrm{cmH}_{2} \mathrm{O}$. The increase in tissue pressure was likely to impact on pulmonary capillaries that represent a network of parallel thin-walled, collapsible channels whose patency depends upon the pressure existing in their perimicrovascular environment, a mechanical behaviour referred to as "Starling resistor" [14]. Thus, it seems reasonable to hypothesise that the increase in peri-microvascular interstitial pressure may represent an important factor to decrease CVI in MA regions; the corresponding increase in regional flow resistance would then divert blood flow towards other zones, similarly to what occurs in oedematous ischaemic brain regions [12]. Moreover, in high-altitude pulmonary oedema (HAPE)-sensitive subjects exposed to hypoxia, a blood shift was found from basal towards the apical regions [15], the former being characteristically more exposed to oedema formation in humans.

\section{Differential vascular adaptive response in WA and MA regions in chronic hypoxia}

The change in volume fraction of the blood reported in figure $2 \mathrm{a}$ may reflect both the increase in $\mathrm{Ht}$ and the change in capillary vascularity. Obviously, the increase in $\mathrm{Ht}$ may account for the increase in the blood volume fraction; yet, the CVI was slightly increased in WA regions, thought not significantly relative to control, mostly due to the increase in corner vascularity.

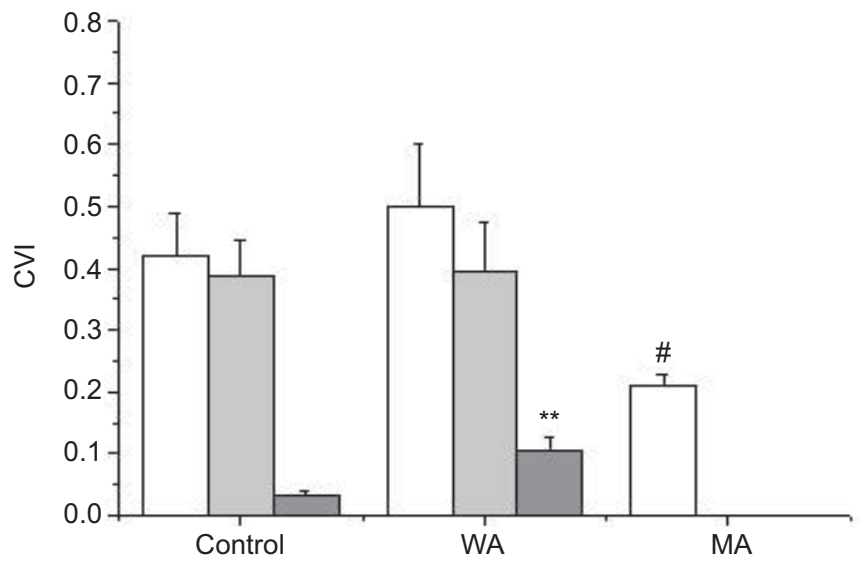

FIGURE 3. Capillary vascularity index (CVI) in control, well-adapted (WA) and maladapted (MA) regions. For control and WA conditions, the index referring to total tissue $(\square)$ was split into septal $(\square)$ and corner $(\square)$ components. ${ }^{* *}$ : $p<0.01$ relative to control; ${ }^{*}$ : $p<0.01$ relative to control and WA.

Conversely, CVI was markedly decreased in MA regions (fig. 3). With regard to the density of pre-capillary vessels of calibre $\leqslant 50 \mu \mathrm{m}$, no appreaciable differences were found in control and WA. Interestingly, the number of vessels in the range 51-100 $\mu \mathrm{m}$ decreased in WA at the expense of the increase in vessels of larger calibre (fig. 4a) that display a decrease in their wall thickness (fig. 4b). Therefore, in the WA regions, the increase in extension in the vascular bed occurred at the capillary and at the pre-capillary level. In the MA regions, the extension of the vascular bed was reduced both at the capillary and pre-capillary levels (figs 3 and $4 a$ ). The data in figure $4 \mathrm{~b}$ show that, for any given internal diameter of the vessels, wall thickness was higher in MA and lower in WA regions, relative to control. An increase in wall thickness may reflect two phenomena: a state of contraction of smooth muscles and/or a proliferative response of myocytes to chronic hypoxia; thus, we reason that the diameter of the vessel would have been larger in absence of either vasoconstriction and/or proliferative response. Therefore, in MA regions, the decrease in the density of the pre-capillary vessels, as well as the increase in thickness/ internal diameter ratio, should imply an increase in vascular resistances. The opposite considerations should apply to WA regions, as a decrease in wall thickness for a given internal diameter might be due either to relaxation or to an antiproliferative response; the functional consequence would be a decrease in vascular resistance. One may interpret the vascular changes in WA and MA regions as the adaptive functional response favouring blood flow distribution to lung regions that still retained diffusion properties [16, 17]. Furthermore, the present data allow us to specifically relate the opposite vascular adaptations in WA or MA zones to the so far unexplained finding of neoangiogenesis and vessel obliteration in the chronic hypoxic lung $[18,19]$.

\section{The propensity to develop pulmonary oedema}

On acute exposure to hypoxia of a comparable level [1] to that of the present study, it was found that the progressive increase in $P$ ip was paralleled by the increase in $P$ pa; the interpretation was put forward that the increase in vasomotor tone acted to 

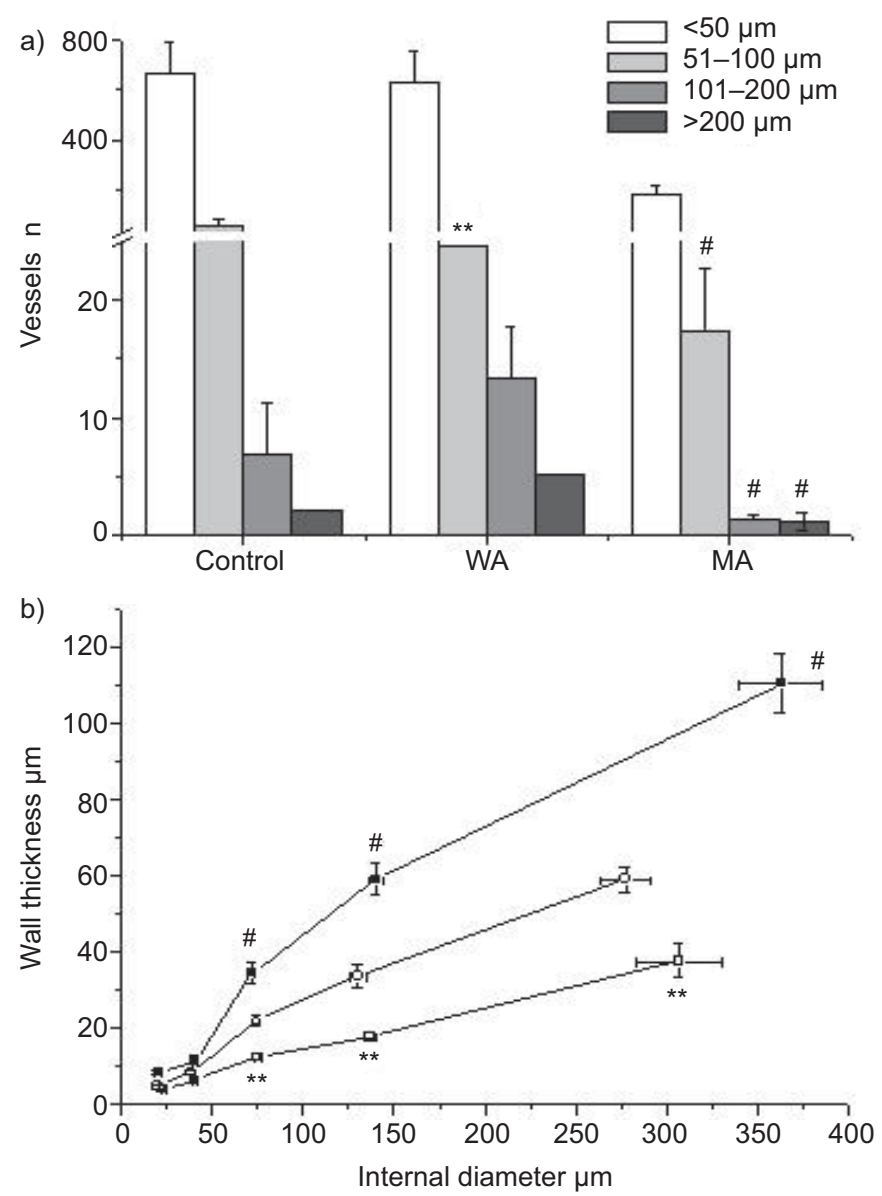

FIGURE 4. a) Density of the pre-capillary vessels grouped into four ranges of internal diameter: in well-adapted (WA) and maladapted (MA) regions relative to control. Density values refer to a surface area of $1 \mathrm{~mm}^{2}$ and were normalised to average tissue volume. b) Thickness of the pre-capillary vessel walls for the internal diameter groups $<30,31-50,51-100,101-200,>200 \mu \mathrm{m}(\mathrm{O}$ : control; $\square$ : WA; n: MA). ${ }^{* *}: p<0.01$ relative to control; ${ }^{*}: p<0.01$ relative to control and WA.

keep hydraulic pressure low in the highly recruited microcirculation $[1,6]$, thus buffering an increase in microvascular filtration.

Furthermore, on acute hypoxia exposure, no major regional differences were found in Pip, suggesting a fairly homogeneous distribution of interstitial oedema within the lung [1], at variance with the present results in chronic hypoxic rats. In the latter, pulmonary hypertension reflects a highly heterogeneous condition in terms of regional distribution of vascular resistances and interstitial tissue pressure. The increase in pulmonary vascular resistances suggests that lung regions where vascular resistances increased prevail over those where vascular resistances decreased. We have ignored so far the real extension of the MA regions, and also the potential contribution of transitional zones, to the increase in pulmonary vascular resistance.

Since interstitial oedema represents an early stage of severe oedema, this poses the critical question of why some regions are more prone to develop severe oedema, while others appear intrinsically more protected against this risk. As oedema formation is strictly related to blood perfusion, one may hypothesise that the heterogeneity in blood perfusion may indeed account for lung regional differences in interstitial fluid dynamics. A recent high-resolution study [20] suggests that the main factors affecting the distribution of pulmonary blood flow may reflect the intrinsic individual topology and geometry of the anatomical arterial vessel: in practice, local vascular resistances. In support of this hypothesis is the finding that an increase in heterogeneity in pulmonary blood flow was found in subjects with a history of HAPE exposed to hypoxia, compared with normal subjects [21].

The question, then, is how can WA regions, which are likely to receive a greater blood flow, resist the development of severe oedema? A more favourable condition to resist oedema formation ought to be interpreted in terms of Starling balance of pressure for transvascular fluid fluxes. One may hypothesise that a specific local lung architectural design, including the geometry of the vascular tree, allows microvascular permeability to remain low. From previous work in our group, we found that hypoxia inhibits caveolar expression [9-11], a condition known to favour the matrix deposition [22]. Thus, one may suggest that in WA regions, local remodelling of the matrix might ensure its integrity providing a long lasting "tissue safety factor".

\section{SUPPORT STATEMENT}

This work was funded by an institutional grant to G. Miserocchi (Fondo di Ateneo per la Ricerca, Università Milano-Bicocca, 2008) and by CV Therapeutics (Palo Alto, CA, USA) to A. Zaza.

\section{STATEMENT OF INTEREST}

Statements of interest for A. Zaza and the study itself can be found at www.erj.ersjournals.com/site/misc/statements.xhtml

\section{REFERENCES}

1 Miserocchi G, Passi A, Negrini D, et al. Pulmonary interstitial pressure and tissue matrix structure in acute hypoxia. Am J Physiol Lung Cell Mol Physiol 2001; 280: L881-L887.

2 Miserocchi G, Negrini D, Gonano C. Direct measurements of interstitial pulmonary pressure in in-situ lung with intact pleural space. J Appl Physiol 1990; 69: 2168-2174.

3 Howard CV, Reed MG. Unbiased Stereology: Three-Dimensional Measurement in Microscopy. New York, Springer-Verlag, 1998.

4 Miserocchi G. Mechanisms controlling the volume of pleural fluid and extravascular lung water. Eur Respir Rev 2009; 18: 244-252.

5 Miserocchi G, Negrini D, Passi A, et al. Development of lung oedema: interstitial fluid dynamics and molecular structure. News Physiol Sci 2001; 16: 66-71.

6 Miserocchi G. Lung interstitial pressure and structure in acute hypoxia. In: Roach RC, Wagner PD, Hackett PH, eds. Hypoxia and the Circulation. New York, Springer, 2007; pp. 141-157.

7 Al Haboubi H, Tomlinson D, Ward B. The influence of hypoxia on transvascular leakage in the isolated rat heart: quantitative and ultrastructural studies. J Physiol 1995; 482: 157-166.

8 Gonzalez NC, Allen J, Gustavo Blanco V, et al. Alveolar macrophages are necessary for the systemic inflammation of acute alveolar hypoxia. J Appl Physiol 2007; 103: 1386-1394.

9 Daffara R, Botto L, Beretta E, et al. Endothelial cells as early sensors of pulmonary interstitial oedema. J Appl Physiol 2004; 97: 1575-1583.

10 Palestini P, Calvi C, Conforti E, et al. Composition, biophysical properties and morphometry of plasma membranes in pulmonary interstitial oedema. Am J Physiol Lung Cell Mol Physiol 2002; 282: L1382-L1390. 
11 Botto L, Beretta E, Daffara R, et al. Biochemical and morphological changes in endothelial cells in response to hypoxic interstitial oedema. Respir Res 2006; 7: 7.

12 Pranevicius M, Pranevicius O. Cerebral venous steal: blood flow diversion with increased tissue pressure. Neurosurgery 2002; 51 : 1267-1274.

13 Nathan SS, Huvos AG, Casas-Ganem JE, et al. Tumor interstitial fluid pressure may regulate angiogenic factors in osteosarcoma. J Orthop Res 2008; 26: 1520-1525.

14 Hopkins S, Garg J, Bolar D, et al. Pulmonary blood flow heterogeneity during hypoxia and high-altitude pulmonary edema. Am J Respir Crit Care Med 2005; 171: 83-87.

15 Permutt S, Riley RL. Hemodynamics of collapsible vessels with tone: the vascular waterfall. J Appl Physiol 1963; 18: 924-932.

16 Capen R, Latham L, Wagner WJ. Diffusing capacity of the lung during hypoxia: role of capillary recruitment. J Appl Physiol 1981; 50: 165-171.
17 Moudgil R, Michelakis ED, Archer SL. Hypoxic pulmonary vasoconstriction. J Appl Physiol 2005; 98: 390-403.

18 Howell K, Preston RJ, McLoughlin P. Chronic hypoxia causes angiogenesis in addition to remodelling in the adult rat pulmonary circulation. J Physiol 2003; 547: 133-145.

19 Stenmark KR, McMurtry IF. Vascular remodeling versus vasoconstriction in chronic hypoxic pulmonary hypertension: a time for reappraisal? Cir Res 2005; 97: 95-98.

20 Hanaoka M, Tanaka M, Ge R, et al. Hypoxia-induced pulmonary blood redistribution in subjects with a history of high-altitude pulmonary edema. Circulation 2000; 101: 1418-1422.

21 Ngeow YK, Mitzner W. Pulmonary hemodynamics and gas exchange properties during progressive edema. J Appl Physiol 1983; 55: 1154-1159.

22 Gosens R, Mutawe M, Martin S, et al. Caveolae and caveolins in the respiratory system. Curr Mol Med 2008; 8: 741-753. 